\title{
DÜBLIN
}

Technological University Dublin

ARROW@TU Dublin

Articles

School of Physics \& Clinical \& Optometric

Science

2007-01-01

\section{Colour Doppler Spatial Resolution Performance Testing}

\author{
Jacinta Browne \\ Technological University Dublin, jacinta.browne@tudublin.ie \\ Ian Brown \\ Western Infirmary Glasgow \\ Peter Hoskins \\ University of Edinburgh
}

See next page for additional authors

Follow this and additional works at: https://arrow.tudublin.ie/scschphyart

Part of the Biological and Chemical Physics Commons

\section{Recommended Citation}

Browne, J., Brown, I., \& Hoskins, P. (2007). Colour Doppler Spatial Resolution Performance Testing. Ultrasound, vol.15, no. 3, pg.162-167. doi:10.1179/174313407X208677

This Article is brought to you for free and open access by the School of Physics \& Clinical \& Optometric Science at ARROW@TU Dublin. It has been accepted for inclusion in Articles by an authorized administrator of ARROW@TU Dublin. For more information, please contact arrow.admin@tudublin.ie, aisling.coyne@tudublin.ie, gerard.connolly@tudublin.ie.

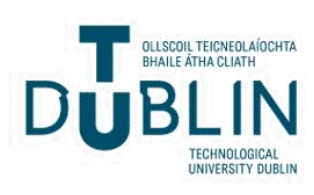




\section{Authors}

Jacinta Browne, lan Brown, Peter Hoskins, Amanda Watson, and Alex Elliott

This article is available at ARROW@TU Dublin: https://arrow.tudublin.ie/scschphyart/9 


\section{COLOUR DOPPLER SPATIAL RESOLUTION PERFORMANCE}

\section{TESTING}

JACINTA E BROWNE ${ }^{1,2}$, IAN BROWN ${ }^{1}$, PETER R HOSKINS ${ }^{3}$, AMANDA J WATSON $^{1}$ AND ALEX T ELLIOTT ${ }^{1}$.

${ }^{1}$ Department of Clinical Physics, Western Infirmary, Glasgow, UK.

${ }^{2}$ School of Physics, Dublin Institute of Technology, Dublin 8, Ireland.

${ }^{3}$ Medical Physics Unit, University of Edinburgh, Edinburgh, UK.

Dr Jacinta Browne,

School of Physics,

Dublin Institute of Technology,

Kevin Street,

Dublin 8,

Ireland.

Email: jacinta.browne@dit.ie

Running Title: Colour Doppler Spatial Resolution

Key Words: Colour Doppler Spatial Resolution; Doppler Ultrasound; Flow Phantom;

Quality control testing. 


\section{COLOUR DOPPLER SPATIAL RESOLUTION PERFORMANCE}

\section{TESTING}

Background: Colour Doppler has become an integral part of many clinical ultrasound investigations, but evaluation of the spatial resolution of colour Doppler scanners remains problematic due to a lack of suitable test phantoms.

Methods: The aims of this study were to evaluate two designs of colour Doppler spatial resolution test-phantom, to compare their suitability for determining the spatial resolution of five commercially available ultrasound scanners. For the purpose of this study, spatial resolution was defined as the minimum resolvable distance between two channels of flow. Phantom 1 consisted of two diverging flow-channels. Phantom 2 consisted of four pairs of parallel channels with different separations.

Results and Discussion: Both designs of phantom highlighted a number of advantages and disadvantages. In principle, phantom 1 allowed the minimal resolvable distance to be identified based on imaging a continuous range of separations, but in practice, the average separation being probed was uncertain, and the minimum separation between vessels of $1 \mathrm{~mm}$ was too large to test the resolutions of some of the scanners we studied. Phantom 2 was easier to set-up and had a narrower minimum separation of $0.6 \mathrm{~mm}$, but only four nominal separations were tested.

Evaluation of five scanners using each prototype phantom suggested typical resolutions of $1.2 \mathrm{~mm}$ or lower. Many of the scanners possessed resolutions of approximately $0.6 \mathrm{~mm}$ or less, which was the minimum separation we were able to 
test. The poorest lateral resolution of approximately $1.2 \mathrm{~mm}$ was observed for a 13 year old scanner with curvilinear $4 \mathrm{MHz}$ phased array transducer.

Conclusion: Construction of the 'perfect' test-object for measuring colour Doppler resolution remains a considerable challenge. Further work will be required to produce acoustically realistic flow phantoms capable of testing the ability of colour Doppler to resolve increasingly narrow flow separations. 


\section{INTRODUCTION}

Continuous and pulsed wave Spectral Doppler and Duplex Doppler imaging modes have been tested for a number of years using well established protocols and test phantoms. Commercially available string phantoms and flow-rigs have been used to test various aspects of Duplex system performance such as maximum velocity estimation. ${ }^{1,2}$ However, despite the existence of recommended performance test protocols for colour and power Doppler imaging, very few test devices existed while this work was carried out. ${ }^{3,4,5,6,7}$ Recently a new research Doppler test device called the DOPHAN (Department of Medical Physics \& Bio-Engineering, St George's Hospital, University of London) has been developed, but the implementation of colour and power Doppler performance testing remains limited.

The current recommended standard for evaluating colour Doppler spatial resolution is measurement of the minimum separation that systems can display between tubes embedded in a tissue-mimicking material through which blood mimicking fluid is pumped. (Draft IEC 2002 87/231/NP: 'Ultrasonics - Colour flow imaging systems - Test procedures to determine performance') However, previous studies have evaluated colour Doppler spatial resolution based on an acoustic grid, an oscillating thin-film, or a modified string phantom consisting of two strings with adjustable separations. ${ }^{5,6,8}$ The acoustic grid measured lateral spatial resolution for separations between $0.5 \mathrm{~mm}$ and $10 \mathrm{~mm},{ }^{6}$ but the arrangement of the acoustic grids had potential to produce diffraction of the ultrasound beam. The modified string phantom provided measures of spatial resolution between 0.5 and $10 \mathrm{~mm},{ }^{5}$ but measurements were carried out in water using O-ring rubber, which has a significantly higher backscatter value than blood. This would have produced a colour Doppler signal that was significantly stronger than typical signals from blood-flow. 
Furthermore, string phantoms are not particularly suitable for measuring colour Doppler spatial resolution, because instead of producing a volume of flow, they produce a narrow line of flow. The third study of colour Doppler spatial resolution was performed by Phillips et al. who constructed an oscillating thin-film test object consisting of a number of precisely deposited sub-resolvable scatters. ${ }^{8}$ Unfortunately, the analysis of the images was time-consuming and not practical for quality control tests in a busy ultrasound department.

The aim of this study was to design and evaluate a simple to use spatial resolution flow phantom based on IEC 2002 87/231/NP recommendations. Two methods were employed to investigate the minimum resolvable separation between two tubes of fluid; the first used a flow phantom containing two diverging vessels of varying separation (phantom 1), while the second contained four pairs of parallel vessels with nominal separations (phantom 2). These phantoms were used to assess the colour Doppler spatial resolutions of five commercial ultrasound scanners, as listed in Table 1.

\section{METHODS}

\section{Phantom 1}

The first phantom contained two identical diverging vessels running parallel to the top of a $15.5 \mathrm{~cm}$ long flow-tank at a constant depth of $2 \mathrm{~cm}$, Fig. 1. Vessels were constructed from poly-tetra-fluro-ethyelene (PTFE) tubing (Cole-Parmer, UK), with internal diameter of $1.6 \mathrm{~mm}$, and speed of sound of $1950 \mathrm{~m} \mathrm{~s}^{-1}$ measured at $25^{\circ} \mathrm{C}^{2}$ The vessels diverged at an angle of $1.7^{\circ}$ producing a continuously varying lateral separation ranging between 1 and $1.6 \mathrm{~mm}( \pm 0.2 \mathrm{~mm})$. The minimum achievable separation was $1 \mathrm{~mm}$ due to the $0.5 \mathrm{~mm}$ thickness of the vessel walls. An acoustic 
marker was placed at one end of the phantom to aid with positioning of the probe. Vessel separations were measured at $1 \mathrm{~cm}$ intervals along the length of the phantom using a Vernier calliper $( \pm 0.03 \mathrm{~mm})$; this resulted in an uncertainty of $\pm 0.2 \mathrm{~mm}$ in each of the spatial resolution measurements due both to repositioning and the effect of the finite slice width. The test phantom was then filled with tissue-mimicking material (TMM), with an attenuation coefficient of $0.5 \mathrm{~dB} \mathrm{~cm}^{-1} \mathrm{MHz}^{-1}$ and speed of sound of $c=1536 \mathrm{~m} \mathrm{~s}^{-1}$, measured at $22^{\circ} \mathrm{C}^{11}$ Blood-mimicking fluid $(\mathrm{BMF})^{9,10}$ was circulated through the tubing using a pump system. ${ }^{12}$

\section{Phantom 2}

The second phantom contained four pairs of nylon vessels with constant separations of $0.6 \pm 0.03 \mathrm{~mm}, 0.8 \pm 0.03 \mathrm{~mm}, 1.0 \pm 0.03 \mathrm{~mm}$ and $1.2 \pm 0.03 \mathrm{~mm}$, Fig. 2. The nylon tubing (Cole-Parmer, UK) had a speed of sound of $2600 \mathrm{~m} \mathrm{~s}^{-1}$ measured at $22^{\circ} \mathrm{C}$. Nylon was chosen because of its narrow wall thickness of $0.3 \mathrm{~mm}$, which produced a minimum separation of $0.6 \mathrm{~mm}$. The vessels had an internal diameter of $1.9 \mathrm{~mm}$ and were orientated at an angle of $45^{\circ}$ to the top of the phantom. The phantom was filled with TMM and blood-mimicking fluid circulated using a pump.

\section{Doppler Colour Flow Imaging Scanners}

Colour Doppler spatial resolution measurements were obtained for five commercial ultrasound scanners equipped with a selection of probes. Scanners were of various costs and ages, varying from over ten years to just a few months old, as described in Table 1. All of the scanners were in routine clinical use within the North Glasgow University NHS Trust during the time of this study. Colour Doppler images were acquired for each of the two flow phantoms using the following test protocol. 


\section{Test protocol}

In both phantoms the transducer was set at an angle of $45^{\circ}$ to the vessels. Phantom 1 was imaged at a depth of $20 \mathrm{~mm}$, while the vessels in phantom 2 were imaged at a depth of $70 \mathrm{~mm}$. Steady-flow of $50 \mathrm{~cm} \mathrm{~s}^{-1}$ was generated by the pump. The phantoms were imaged transversely using B-mode imaging, which was optimised by adjusting the 2D gain and time-gain controls. Following optimisation, the colour Doppler function was enabled and optimised by adjusting the size of the colour Doppler box, position of the focal zone, and gain. The colour scale was set with the highest velocity of the scale just above the true maximum velocity of the flow. The colour gain was adjusted so that the colour image filled the vessels, without colour leakage into the vessel wall or the surrounding medium. The minimum detectable lateral separation was then determined from colour Doppler images of flow-phantom line-pairs.

Images were obtained five times to determine a $95 \%$ C.I. associated with each measurement. A CT scan of phantom 2 was also used to verify that the separation between the four individual pairs of vessels remained constant with depth. In phantom 1 , there were uncertainties in average minimum separation of $\pm 0.2 \mathrm{~mm}$, due to difficulties in correlating the sample volume with the corresponding separation at that point in the tubing. Slight changes in separation also occurred across the slice width. Experimental uncertainties in phantom 2 correspond to the difference between minimum distances between each set of lines $( \pm 0.2 \mathrm{~mm})$.

Instrument parameters including focal-depth, persistence, colour Doppler gain and B-mode gain, were then varied to determine the effects of instrument settings on the lowest measurable separation. 


\section{RESULTS}

\section{Comparison of scanners}

The minimum resolvable separation of flow channels was investigated using both phantoms for five ultrasound scanners, Table 1. Scanners C and D both had resolutions that were better than the $0.6 \mathrm{~mm}$ minimum separation that could be measured by phantom 2. The oldest scanner (scanner E) had the poorest lateral resolution, estimated at $1.2 \mathrm{~mm} \pm 0.2 \mathrm{~mm}$. Example images produced by scanner $\mathrm{C}$ and scanner E are presented in Fig. 3.

\section{The Effect of Instrument Settings}

All instrument settings that were investigated had an effect on colour Doppler lateral resolution, Fig. 4. Colour Doppler gain and persistence had a marked effect on subjective visualisation of the flow phantom vessels. This is demonstrated for scanner D with curvilinear 1-4 MHz transducer in Figs. 4 (a) and (b). The effects of both Bmode gain and focal point position on the visualisation of the flow phantom vessels using the same scanner and transducer are highlighted in Figs. 4 (c) and (d). Our results from a previous study ${ }^{13}$ showed a moderate correlation between the technical assessment of colour Doppler lateral resolution and the clinical perception of colour Doppler resolution $\left(R^{2}=-0.4, p<0.05\right)$.

\section{DISCUSSION}

The colour Doppler spatial resolution of five ultrasound scanners was investigated by measuring the minimum separation detectable in two designs of prototype flowphantom. Phantom 1 contained a pair of diverging vessels producing a continuous variation in vessel separation ranging between 1.0 and $1.6 \mathrm{~mm}$. However, difficulties 
in correlating the position of the sample volume with the minimum separation at that point in the phantom, as well as slight changes in separation over the width of the slice ( $\pm 0.2 \mathrm{~mm}$ for a $45^{\circ}$ angle of insonation and slice thickness of $3 \mathrm{~mm}$ ), mean that the uncertainty in the estimated minimum separation being probed was around \pm 0.2 $\mathrm{mm}$. This could be improved in future phantoms by introducing acoustic markers at regular intervals along the length of the vessels, and by lengthening the phantom to render the effects of slice thickness negligible.

Phantom 2 contained four pairs of parallel vessels with separations ranging from $0.6 \mathrm{~mm}$ to $1.2 \mathrm{~mm}$ in $0.2 \mathrm{~mm}$ increments. Minimum separations in both phantoms were limited by vessel wall thicknesses of $0.5 \mathrm{~mm}$ for phantom 1 and $0.3 \mathrm{~mm}$ for phantom 2. Although phantom 2 presented a lower minimum separation of $0.6 \mathrm{~mm}$, measurements were confined to discrete distances. Resolutions were therefore either $<0.6 \mathrm{~mm}, 0.6-0.8 \mathrm{~mm}, 0.8-1.0 \mathrm{~mm}, 1.0-1.2 \mathrm{~mm}$, or $>1.2 \mathrm{~mm}$. For example, a system capable of resolving a $0.8 \mathrm{~mm}$ separation but not a $0.6 \mathrm{~mm}$ separation can be concluded as possessing a resolution lying somewhere between 0.6 and $0.8 \mathrm{~mm}$. Phantom 2 was of a less sophisticated design, but was easier to use than phantom 1 . The $45^{\circ}$ angle of the tubes was advantageous for orienting the probe, and the results were found to be useful for placing limits on colour Doppler resolution.

A significant problem with both phantoms was that the vessels were constructed from tubing that produced significant attenuation of the ultrasound beam. Shadowing can be seen in Figs. 3 and 4. Different vessel materials with lower attenuation are currently being sought to alleviate this problem.

The effects of variations in instrument settings were also investigated, Fig. 4. Colour gain had a marked effect on the visualisation of the colour Doppler signal; insufficient gain caused the signal at the edge of the vessels to be removed, whereas 
excessive gain caused the signal to bleed over into the area occupied by the vessel walls. The effects of focal-zone depth and persistence were also pronounced. The optimum depth of focal-zone was found to be beside the vessel. The optimum setting for persistence was always the highest setting. B-mode gain slightly affected the visualisation of the colour Doppler signal in the vessels, with both insufficient and excessive gain causing loss of signal at the edge of the vessel.

Doppler frequency also had a marked effect on Doppler spatial resolution performance. Similar trends for colour Doppler were found by Li et al. ${ }^{6}$ when varying colour Doppler instrument settings and experimental conditions. It should be noted that neither of the spatial resolution flow phantoms tested here would be suitable for determining the colour Doppler spatial resolution of transducers with Doppler frequencies higher than $5 \mathrm{MHz}$, because the scan resolution would better than the minimum separation of the vessels. All of the linear probes tested had spatial resolutions that were better than the minimum separation tested by our phantoms.

\section{CONCLUSIONS}

In summary, flow-phantom testing of colour Doppler spatial resolution makes it possible to perform a simple assessment of colour Doppler imaging resolution for a range of ultrasound scanners. Both phantoms we tested provided a means of determining the performances of ultrasound systems of differing cost and complexity. Our approach was based on measuring the minimum resolvable separation between lines of flow produced by two prototype spatial resolution flow-phantoms. The designs of the phantoms incorporated diverging vessels and parallel pairs of vessels with different separations. Both of the phantoms had different advantages and limitations, which will be incorporated into the design of future test-phantoms. 
Our investigation revealed the effects of instrument settings on the resultant colour Doppler image. Although our results were predominantly qualitative they indicate that care needs to be taken during selection of image and colour Doppler settings if optimum colour Doppler resolution is to be obtained.

Our results demonstrate that suitable flow-phantom test objects could in principle provide quick and useful measurements of colour Doppler imaging resolution performance. Creation of a 'perfect' Doppler spatial resolution test-object will require careful selection of the optimum geometry of the tubes. Further work is also required to improve the acoustic properties of test phantoms, and to reduce minimum test separations using tubing with narrow wall thickness. 


\section{REFERENCES}

1. Hoskins PR. Choice of Moving Target for A String Phantom .1. Measurement of Filament Backscatter Characteristics. Ultrasound in Medicine and Biology. 1994;20:773-780.

2. Hoskins PR, Sherriff SB, and Evans JA. Testing Doppler Ultrasound Equipment. 1994, IPSM Report No 70.

3. Deane CR, Forsberg F, Thomas N, and Roberts VC. Accuracy of Color Doppler Ultrasound Velocity-Measurements in Small Vessels. Journal of Biomedical Engineering. 1991;13:249-254.

4. Goldstein A. Performance Tests of Doppler Ultrasound Equipment with A String Phantom. Journal of Ultrasound in Medicine. 1991;10:125-139.

5. Lange GJ and Loupas T. Spectral and color Doppler sonographic applications of a new test object with adjustable moving target spacing. Journal of Ultrasound in Medicine. 1996;15:775-784.

6. Li S, Hoskins PR, and McDicken WN. Rapid measurement of the spatial resolution of colour flow scanners. Ultrasound in Medicine and Biology. 1997;23:591-596.

7. Rickey DW and Fenster A. A Doppler ultrasound clutter phantom. Ultrasound in Medicine and Biology. 1996;22:747-766.

8. Phillips D, McAleavey S, and Parker K. Colour Doppler Spatial Resolution Measurements with an Oscillating Thin Film Test Object. IEEE Ultrasonics Symposium. 1997;1517-1520.

9. Ramnarine KV, Hoskins PR, Routh HF, and Davidson F. Doppler backscatter properties of a blood-mimicking fluid for Doppler performance assessment. Ultrasound in Medicine and Biology. 1999;25:105-110. 
10. Ramnarine KV, Nassiri DK, Hoskins PR, and Lubbers J. Validation of a new blood-mimicking fluid for use in Doppler flow test objects. Ultrasound in Medicine and Biology. 1998;24:451-459.

11. Teirlinck CJPM, Bezemer RA, Kollmann C, Lubbers J, Hoskins PR, Ramnarine KV, Fish P, Fredfeldt KE, and Schaarschmidt UG. Development of an example flow test object and comparison of five of these test objects, constructed in various laboratories. Ultrasonics. 1998;36:653-660.

12. Browne JE, Watson AJ, Hoskins PR, and Elliott AT. Validation of a sensitivity performance index test protocol and evaluation of colour Doppler sensitivity for a range of ultrasound scanners. Ultrasound in Medicine and Biology. 2004;30:14751483.

13. Browne JE, Watson AJ, Muir C, Hoskins PR and Elliott AT. An investigation of the relationship between in-vitro and in-vivo ultrasound image quality parameters. Ultrasound. 2004;12: 202 - 210. 
Table 1: Summary of ultrasound scanners with results from phantoms 1 and 2.

\begin{tabular}{|c|c|c|c|c|c|c|c|c|}
\hline \multirow[t]{2}{*}{ Scanner } & \multirow[t]{2}{*}{ Age } & \multirow[t]{2}{*}{$\begin{array}{l}\text { Approx. } \\
\text { cost- when } \\
\text { purchased }\end{array}$} & \multirow[t]{2}{*}{$\begin{array}{c}\text { Probe } \\
\text { (Nominal frequency) }\end{array}$} & \multirow{2}{*}{$\begin{array}{c}\text { Phantom } 1 \\
\text { minimum } \\
\text { resolvable } \\
\text { separation } \\
(\mathrm{mm})\end{array}$} & \multicolumn{4}{|c|}{$\begin{array}{c}\text { Phantom 2 } \\
\text { minimum resolvable } \\
\text { separation }(\mathrm{mm})\end{array}$} \\
\hline & & & & & 0.6 & 0.8 & 1.0 & 1.2 \\
\hline $\boldsymbol{A}$ & New & $£ 45,000$ & Curvilinear 2-5 MHz & $1.4 \pm 0.2$ & & & $\checkmark$ & $\checkmark$ \\
\hline $\boldsymbol{B}$ & 1 year & $£ 25,000$ & Curvilinear $2-5 \mathrm{MHz}$ & $1.4 \pm 0.2$ & & $\checkmark$ & $\checkmark$ & $\checkmark$ \\
\hline$C$ & $\begin{array}{c}3 \\
\text { years }\end{array}$ & $£ 100,000$ & $\begin{array}{c}\text { Curvilinear } 2-5 \mathrm{MHz} \\
\text { Linear } 4-7 \mathrm{MHz}\end{array}$ & $\begin{array}{l}<1.0 \\
<1.0\end{array}$ & $\begin{array}{l}\checkmark \\
\checkmark\end{array}$ & $\checkmark$ & $\begin{array}{l}\checkmark \\
\checkmark\end{array}$ & $\begin{array}{l}\checkmark \\
\checkmark\end{array}$ \\
\hline$D$ & $\begin{array}{c}4 \\
\text { years }\end{array}$ & $£ 80,000$ & $\begin{array}{c}\text { Curvilinear } 1-4 \mathrm{MHz} \\
\text { Linear } 5-7 \mathrm{MHz}\end{array}$ & $\begin{array}{c}1.2 \pm 0.2 \\
<1.0\end{array}$ & $\begin{array}{l}\checkmark \\
\checkmark \\
\end{array}$ & $\begin{array}{l}\checkmark \\
\checkmark \\
\end{array}$ & $\checkmark$ & $\begin{array}{l}\checkmark \\
\checkmark\end{array}$ \\
\hline$E$ & $\begin{array}{c}13 \\
\text { years }\end{array}$ & $£ 40,000$ & $\begin{array}{c}\text { Linear phased array } \\
(3.5 \mathrm{MHz})\end{array}$ & $1.6 \pm 0.2$ & & & & $\checkmark$ \\
\hline
\end{tabular}


Figure 1: Cross-sectional plan view of phantom 1.

Figure 2: Photograph of phantom 2.

Figure 3: Colour Doppler images of phantom 2 for (a) scanner E, and (b) scanner $C$ equipped with a curvilinear 2-5 MHz transducer.

Figure 4: Effects of instrument settings on colour Doppler images of phantom 1. (a) Colour gain: (i) insufficient gain, (ii) correct gain, and (iii) excessive gain, (b) Persistence: (i) low persistence value $=1$, (ii) mid persistence $=2$, and (ii) strong persistence $=3$. (c) B-mode gain: (i) insufficient gain, (ii) correct gain, and (iii) excessive gain. (d) Focal Depth: (i) near transducer face, (ii) at the same depth as the vessel, and (iii) at the bottom of the field of view.

Figure 5: Correlation between the technical assessment of colour Doppler lateral resolution and the clinical perception of colour Doppler resolution (phantom 2). 